\title{
34
}

\section{Solution of the problem for a large scale integrated plan in the iron and steel production control}

\author{
Mistushige Shiota and Sumihiro Manabe \\ Nippon Steel Corporation Group \\ 2-8-9 Mitsu-sadadai, Yahatanisiku \\ Kitakyuusyu 807, Japan \\ Tel: +81-93-603-6092 Fax: +81-93-661-5554 \\ E-mail:14330423@people.or.jp
}

\begin{abstract}
The main aim of this report is to explain the production scheduling system in the iron and steel industry which has been developed as part of the consistent systems of sales-production-distribution in the field of sheet-steel production. At the same time, the key points for the solution and practical utilization of the production scheduling system will be outlined.
\end{abstract}

Production scheduling.

Keywords

Man-machine cooperative processes. Iron and steel industry. 


\section{INTRODUCTION(background/target)}

In recent years, in the iron and steel industry as well as in the field of other manufacturing industries facing diversification of orders concerning high grade products with low price, it is indispensable to reduce production costs, shorten the production lead time and secure high quality. It is important to be able to produce a large variety of ordered items, even though the amount may be small, and to supply the customer with fast delivery. Under such circumstances, the production scheduling plays a very important role.

In the proceeding of planning for production, already a precise production scheduling has to be worked out, which then has to be adjusted to every step in order to reach an optimal consistent production scheduling. The conventional batch process system which was used for a long time showed severe difficulties concerning its flexibility - such as changes in the equipment or changes concerning the ordered items and so on.

On the basis of the above mentioned problems, the authors constructed a production scheduling system characterized by the consistent production scheduling from the production of the basic material down to each production step and secondly characterized by the man-machine cooperative processes.

\section{OUTLINE OF THE PRODUCTIION CONTROL IN THE YAWATA IRON AND STEEL MAKING PLANT}

\subsection{Characteristics of the production structure}

The iron and steel production structure follows the "multistage manufacturing process" composed of the large scale batch type equipment. Many sorts of molten steel are made from the same molten iron, which then will be subdivided into various intermediate products, using the "break-down" (fragmentation) process, and will finally be changed into the products of the orders. Even though each production process is adaptable to severe chance conditions (rearrangement condition), it is desirable that a large amount can be produced under the same condition.

A minimum of operation for rearrangement is desirable for the production and distribution control concerning the multistage manufacturing process including the facilities with severe chance conditions concerning a high standard of quality and a reduction of the production costs. For such reasons, in-between each processing step a selection of items is gathered for all products that can follow the same mode of production. Such buffers for integration are used for each step. 


\subsection{Production control system}

In the Yawata iron and steel making plant, on-line computer systems are used for the sheet steel production and the field control for the products includes the collection of the actual records and processing instructions to the filed.

However, the production scheduling control (preparation and revision of the production schedule) is almost conducted by manpower, and the staff in charge of scheduling each process is occupied only by the production scheduling control for the process they are in charge of, so that they can not afford to carry out the scheduling control for the consistent process. For this reason, the production scheduling control can only be a "production control according to the inventory between two processes". This conduction follows the form the "total frame control" in order to balance the production amount in each process. In addition, orders with severe delivery conditions are specifically controlled and be positioned for exceptional control (Figure1).

\subsection{Challenge}

The correspondence to many various kinds of items and the production of only small amounts in the form the present production control will cause an enormous amount of inventory (buffer) between the processes and will lead to a raise in production costs.

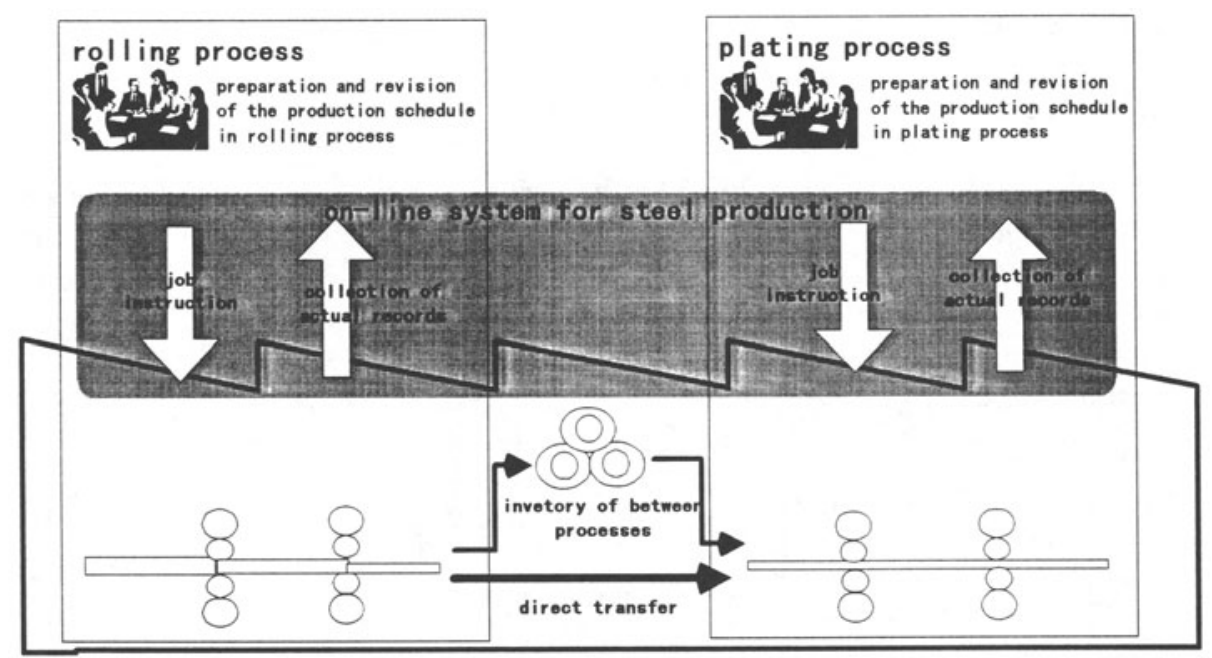

Figure 1 Example of the present production control in the Yawata iron and steel making plant 
Therefore, in order to correspond to a large variety of orders and small quantities at the lowest possible production costs it is essential to reach an ability to plan an optimal production schedule for the consistent process and to realize a flexibility concerning the variations of the environment and constraint during manufacture. Correct timing for necessary rescheduling is very important.

\section{CHARACTERISICS AND SOLUTION OF THE PROBLEMS FOR THE IRON AND STEEL PRODUCTION CONTROL}

\subsection{Characteristics of the problems}

In order to solve the above mentioned problems, it is necessary to assume the processes between the steel making, hot rolling and sheet steel processes as a continuous and consistent process and to plan the consistent schedule in consideration of the constraint conditions such as the passage constraint, the equipment constraint, and so on. for the total material resources.

However according to out judgment that it is unrealistic to solve the complex and large scale combined optimization problem including complex evaluating function, the constraint condition and the large scale material resource as one large scale problem, we have decided to divide the problem into partial problems and to combine the structures in order to get a total optimal solution aiming at the whole conformity by considering each optimal solution.

\subsection{Solution of the problem}

\subsubsection{Structure of the solution}

The large scale combined problem has been divided into three partial problems as follows (Figure2).

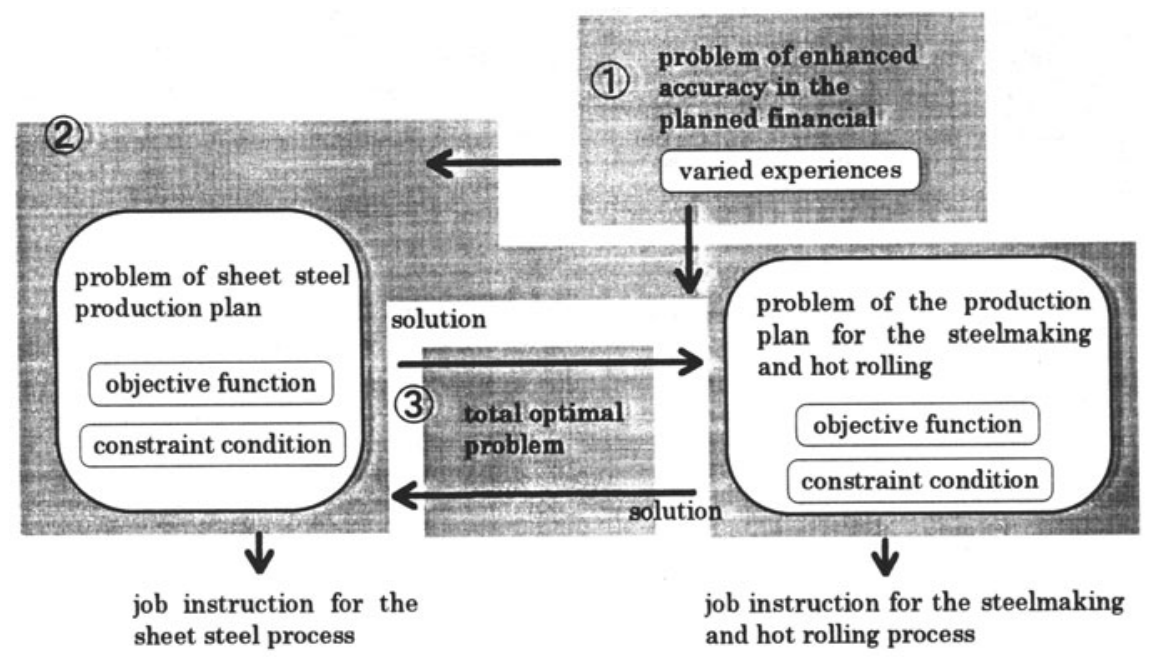

Figure 2 Structure of solution 
1. Problem of enhanced accuracy concerning the material resource for planning

As shown in the previous chapter, in the conventional planning the flow frame (roughly classified material resource for order) was treated as one unit. Therefore, the plan was very rough and will fall far short of the realistic plan, even after the consistent plan is made. The more consistence is aimed at, the more important the problem becomes.

2. Problem of the partial planning for steel making, hot rolling and sheet steel production planning.

A production plan will be made for each of the steel making, the hot rolling and the sheet steel processes concerning the accurate material resource. More than in previous times, concerning the development of the algorithm aiming at the enhanced operation rate for equipment with consideration of the equipment constraint and the passage constraint, etc., the flexibility of the plan still remains as a very important point. A flexible process adjustable to the circumstances has to aim at a suitable arrangement for the best harmony with human beings.

3. Total optimal problem.

The total optimality is oriented by incorporating the solution of the above mentioned problem as the mutual conditions of each algorithm.

Because these processes correspond to varied actual records, the system needs daily planning. The content of the measure for each problem is briefly explained as follows.

\subsubsection{Measure of enhanced accuracy concerning the material resources for planning.}

Assuming the material resource of the order as the virtual product, the material resource is produced accurately - reflected by the precise data, the production amount and by the daily varying actual records, which can be produced.

Utilizing the temporary manufacturing information used for the material design in the previous process, the material resource has been produced with additional information which is used for planning. In this system, the temporary manufacturing information has been defined as a design (virtual) production information (Figure3).

Also in the real product information, the varied information in the daily plant operation, that is precisely caught, is reflected to the material resource serving as a basis for planning. 


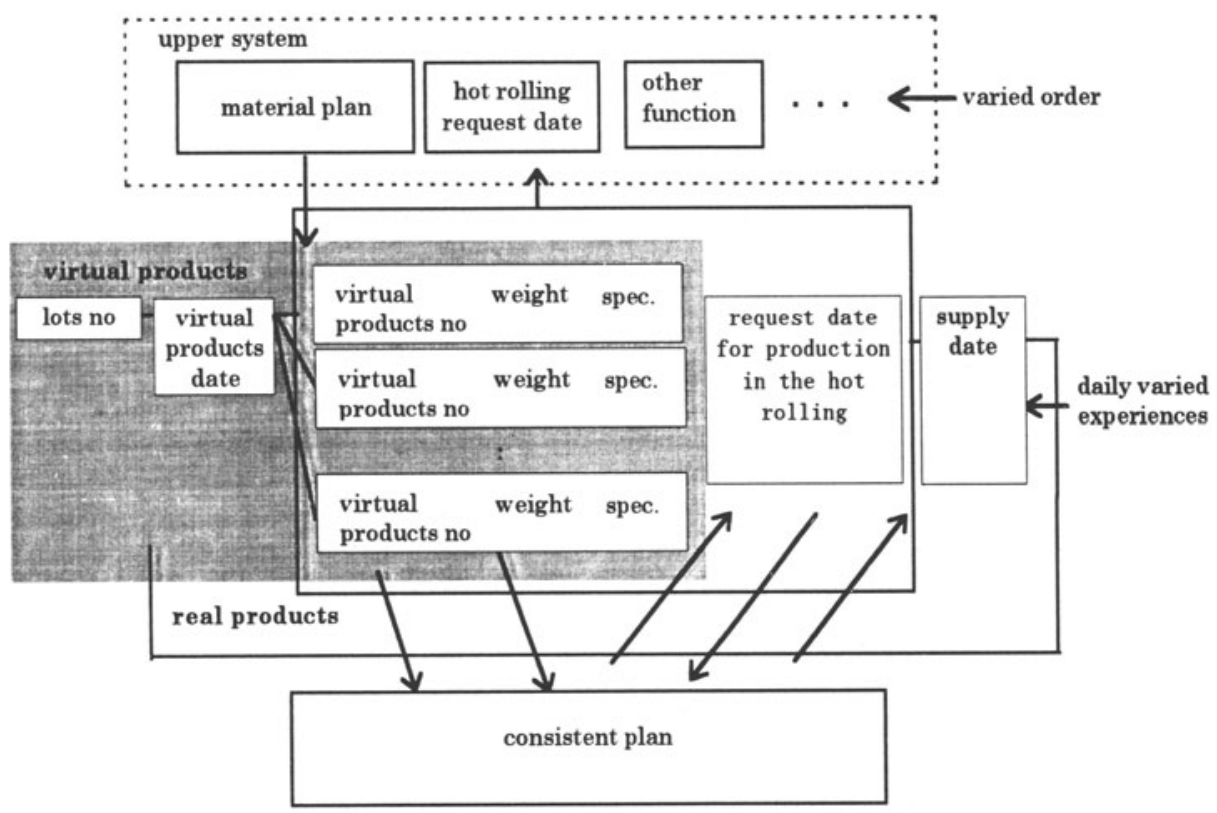

Figure 3 Virtual production information

\subsubsection{Solution of the partial problem (problem of the steel making and hot rolling as well as the sheet steel production planning).}

Aiming at the optimality of each independent plant operation in the steel making, hot rolling and sheet steel process, the development has been conducted as follows.

1. Development of the schedule algorithm

a. Outline of the schedule algorithm for sheet steel The planning problem is formulated as follows.

Objective function : Equipment operation ratio

Constraint condition: Delivery limits of the orders, plant capacity (production capacity, yard capacity)

Though the problem was divided, the scale of the material resource is great. In our case, the number of processing lines for sheet steel is 58 and the material resources are about 10,000 units. 
Further, on account of the daily processes, the operation time for solution is constrained to a large extent and the problem is too difficult to be solved as a simply combined problem. The following algorithm is administered for the early solution of the problem.

- Arrangement of the material resource along the delivery-limit information

- Accumulation of the total schedule - starting from the lowest production capacity processes.

- Arrangement of the supply-demand balance for material between processes by means of the recurrence method.

b. Outline of the schedule algorithm for the steel making and hot rolling

As different from the problem of the sheet steel process planning, the delivery time - (because it is not an order delivery but a manufacturing delivery) - has not been included in the constraint condition but is arranged as an evaluating function.

[formulation]

Objective function: Equipment operation ratio and sheet steel request delivery

Constraint condition : Plant capacity (production capacity / yard capacity)

This algorithm is heuristic, and the following device is administered for the early solution of the problem.

- Preparation/application of the standard pattern with schedule arrangement

- Setting of the priority condition for each process (priority of production efficiency, priority of production capacity, priority of special steel, etc.)

2. Process in harmony with manpower

The key point of this process is GUI. Further, the visualization of the schedule result on the Gnat-chart and the interface with operational excellence, are constructed.

Using this interface, the schedule can be simply revised and evaluated.

In the meeting place where a plurality of personnel in charge can gather at a time for discussion, a large format drawing with the so-called 120-inches is set up. Figure4 indicates the operational procedure of the system. 
large size screen

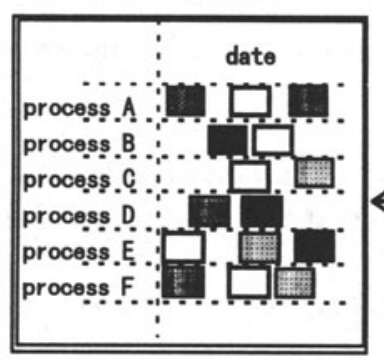

(3) discussion of re-arrangement
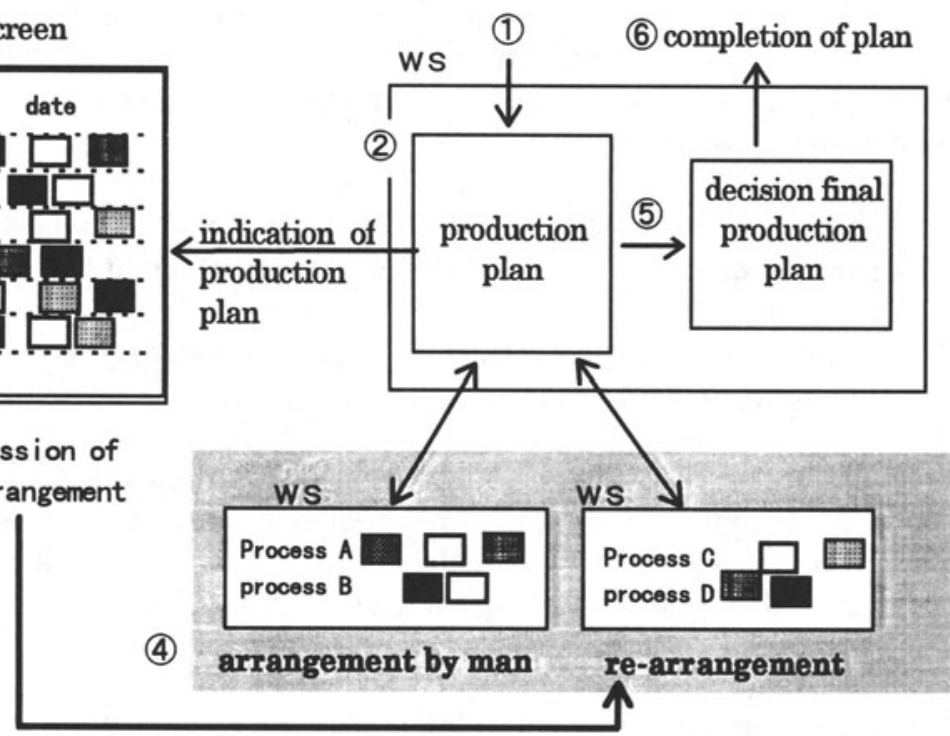

Figure 4 Operational procedure of the system

\subsubsection{The total optimal solution}

The solution of both planning problems is incorporated into the mutual constraint condition in order to get the whole optimization. To be more specific, the depot for the steel making and hot rolling process as well as for the sheet steel process is the exact time schedule for each coil, and the procedure is as follows:

Step1: problem of the sheet steel plan

Step2: problem of the steel making and hot rolling plan after the problem of the sheet steel plan is solved and registered.

Step3: problem of the sheet steel plan after the problem of the steel making and hot rolling plans has been solved and registered.

With respect to the operation instruction, daily operation instructions are published for each plan of the individual process, and it concludes that the operation is conducted according to these instructions (Figure5). 


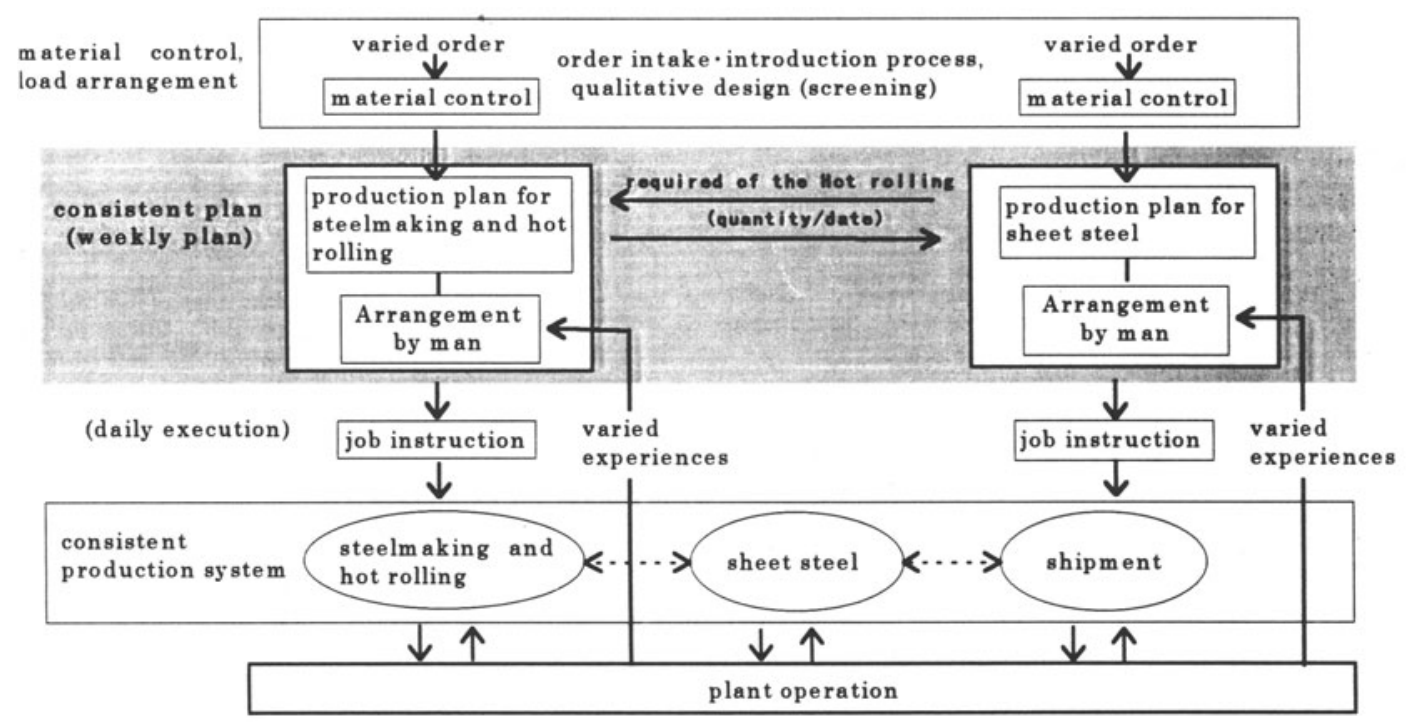

Figure 5 Total optimal solution

\section{COMPUTER SYSTEM}

In the computer technology, we developed our technology so that it can serve as a basis of various kinds of distribution technologies, including the development of the memory control system for high performance of algorithm, development of the cooperative distribution for the cooperation with human beings, development of the remote and centralized monitoring system for the WS, and so on.

\section{APPLICATION EFFECT}

This system is now in operation in the Yawata iron and steel making plant of Nippon Steel Corporation. At present, it is under the evaluation of the introduction effect, but is largely contributed to the administrative challenge, such as the shortened term for planning, reduced inventory, and so on. 


\section{CONCLUSION}

The example of the consistent production plan in the iron and steel industry has been introduced. The key point of this system is that the large scale consistent planning problem has been divided into the partial problems, and further, the partial problems have been arranged with the cooperative system with human being.

We have respected the superiority of the human ability to assess the circumstances over the computer and arranged each system as a cooperative system. In the future, we are thinking about the application of GA(Genetic Algorithm) technology transporting the decision making ability according to the circumstances to the computer in order to reduce the burden on humans.

It seems to be important that the effect of this planning system leads to an understanding of the main (on-line) system which is collecting the actual records and another very important point is the plant operation technology which ensures an exact realization in the proceedings.

We shall be happy if this report is of a help toward the construction of more effective production control systems in the future.

\section{REFERENCE}

Hiroshi KONNO, Hisatoshi SUZUKI : Integer programming and combined optimization. OR library, 1982.(in Japanese)

Kei NAGAI, Kyoji TAMURA, Masayuki NAKAYAMA, Takashi TANIZAKI, Hiroshi NAKAJIMA : Construction of the man-machine cooperative type scheduling system with distributed environment, Communication of the Operations Research Society of Japan, May 1995. (in Japanese)

\section{BIOGRAPHY}

Mitsushige Shiota is General Manager at Logistics Division, Nippon Steel Corporation Group. He received B.E., M.E. in Applied Mathematics and Physics from Kyoto University, Kyoto, Japan, in 1966 and in 1968. His areas of interests are applications of $\mathrm{OR}$, logistics and production control.

Sumihiro Manabe is Group Manager at Computer System Department, Nippon Steel Corporation, Yawata Works. He received B.E., M.E. in Production System from Toyohashi University Technology, Toyohashi, Japan, in 1978 and in 1982. His areas of interests are applications of OR, System technical engineering and production control. 